\title{
Double trouble: a case of von Recklinghausen's disease with coexistent lepromatous leprosy
}

\author{
Kaliaperumal Karthikeyan ${ }^{1}$, Kaliaperumal Latchoumibady ${ }^{2}$, Srinivasan Sowmya ${ }^{3}$ \\ Department of Dermatology and Venereology ${ }^{1}$, Department of Orthopedics ${ }^{2}$, and Department of Pathology ${ }^{3}$, \\ Manakula Vinayagar Medical College, Kalitheerthalkuppam, Pondicherry, India
}

\begin{abstract}
Leprosy (or Hansen disease) is a tropical disease which affects the skin and nerves leading to deformities and disabilities. Von Recklinghausen's disease (neurofibromatosis 1) is a well-recognized genetic disorder with both cutaneous and systemic manifestations. Lesions of neurofibromatosis may be mistaken for nodules of lepromatous leprosy and vice versa. Occurrence of both von Recklinghausen's disease and lepromatous leprosy together is very rare. Since cutaneous nodules can also occur in neurofibromatosis, lepromatous leprosy can be easily missed. The presence of lepromatous leprosy in a patient with von Recklinghausen's disease is unique and poses certain diagnostic dilemmas which are discussed in this report.
\end{abstract}

Key words: leprosy; neurofibromatosis; NF1; von Recklinghausen's disease

J Infect Dev Ctries 2012; 6(3):287-289.

(Received 14 July 2010 - Accepted 18 October 2010)

Copyright $(0) 2012$ Karthikeyan et al. This is an open-access article distributed under the Creative Commons Attribution License, which permits unrestricted use, distribution, and reproduction in any medium, provided the original work is properly cited.

\section{Introduction}

Von Recklinghausen's disease (Neurofibromatosis 1; NF1) is a well-recognized genetic disorder with both cutaneous and systemic manifestation [1]. The neurofibromas seen in this disorder may closely resemble a lepromatous nodule. Leprosy is a tropical disease which affects the skin and nerves leading to deformities and disabilities. The presence of lepromatous leprosy in a patient with von Recklinghausen's disease is unique and poses certain diagnostic dilemmas which are discussed in this report.

\section{Case report}

A twenty-year-old male presented with history of multiple painless cutaneous nodules all over the body for the past six years. The patient also had an associated deformity of the left leg. He had no other systemic complaints. His father also had a similar illness. General physical examination revealed mild pallor. His systemic examination was otherwise normal.

Cutaneous examination revealed multiple hyperpigmented well-defined macules all over the body. He also had numerous cutaneous and subcutaneous nodules on the trunk and the extremities (Figure 1). These nodules demonstrated characteristic "buttonholing"; that is, the application of pressure to these lesions caused them to herniate into the dermis. A plexiform swelling covering the whole of the instep of the left foot was present. The palms, feet and axilla all showed freckling. His left leg was deformed and curved. An orthopedic consultation revealed the presence of pseudoarthrosis of the left tibia. Further detailed examination revealed that the patient had superciliary madarosis and infiltration of the earlobes with multiple colored nodules (Figure 2). His face also showed diffuse infiltration. He had bilateral symmetrical thickening of the supra orbital, greater auricular, ulnar, common peroneal, and posterior tibial nerves. He also had glove and stocking anesthesia of both hands and feet. A clinical diagnosis of neurofibromatosis with coexistent lepromatous leprosy was made.

All his routine investigations were normal except for anemia. A slit skin smear investigation from the nodules and normal-appearing skin showed abundant acid-fast bacilli. The bacteriological index was $6+$. Skin biopsy from the subcutaneous nodules showed features of neurofibroma. Histopathological examination of the nodule on the ear showed epidermal atrophy with diffuse infiltration of the epidermis with histiocytes and a subepidermal Grenz 
Figure 1. Back of the patient showing neurofibromas and café au lait macules

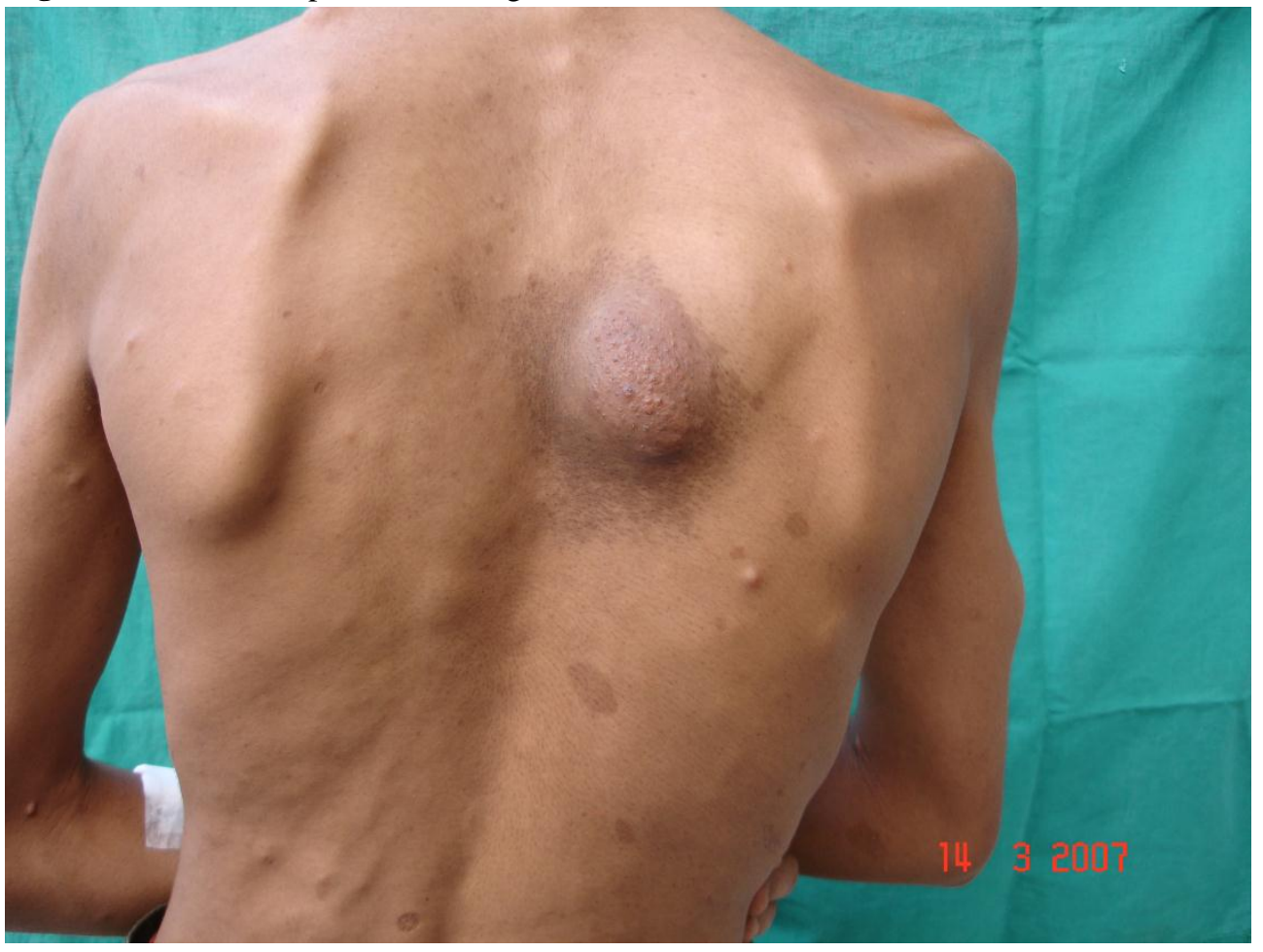

Figure 2. Infiltration and nodules on the ear

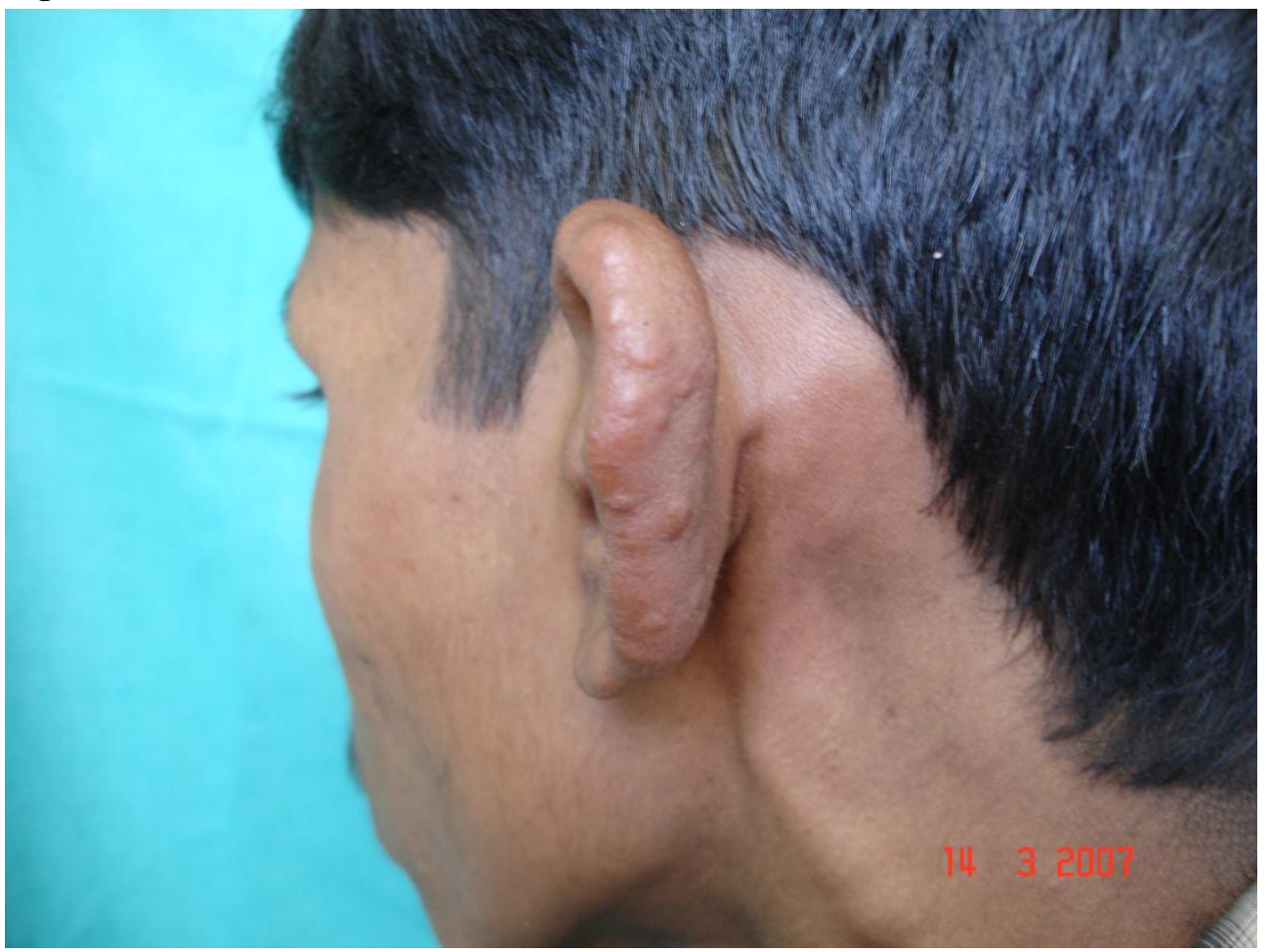


zone. Diagnosis of neurofibromatosis with lepromatous leprosy was made. The patient was started on multidrug therapy recommended by the World Health Organization for multibacillary leprosy. The patient is on follow-up.

\section{Discussion}

Von Recklinghausen's disease is a unique disorder characterized by cutaneous neurofibromas and other cutaneous and extra-cutaneous manifestations [1]. Both leprosy and von Recklinghausen's disease (neurofibromatosis type 1) are diseases affecting the Schwann cells of the peripheral nerves, though both are quite different entities. Lesions of neurofibromatosis may be mistaken for nodules of lepromatous leprosy and vice versa. Occurrence of both von Recklinghausen's disease and lepromatous leprosy together is unique. Since cutaneous nodules can also occur in neurofibromatosis, lepromatous leprosy can be easily missed. Moreover, the features of von Recklinghausen's disease are more prominent and diagnostic than the changes of lepromatous leprosy. The cutaneous neurofibromas that are seen in this disease can easily be distinguished from lepromatous nodules by means of pathognomic features such as the buttonholing sign. Moreover, other features such as café-au-lait macules, Leisch nodules, pseudoarthrosis, and axillary freckling help in distinguishing among these disorders.

Presence of certain features such as infiltration and nodules on earlobes, superciliary madrosis, and diffuse cutaneous infiltration made us suspect coexistent lepromatous leprosy in our patient which was later confirmed by the slit skin smear examination. Neurofibromatosis has been associated with tuberculoid [2], histoid [3] and lepromatous leprosy [4]. Previously only one case of neurofibromatosis and lepromatous leprosy [4] has been reported.

Neurofibromatosis can cause great psychological stress on the patient and presence of leprosy further complicates the problem. In our patient at least one of his diseases can be treated.

Further, in this era of leprosy elimination, it is important to identify every case of leprosy, especially a patient with lepromatous leprosy. Hence it becomes imperative in leprosy endemic countries to look for and rule out leprosy in all disorders which can mimic leprosy-like neurofibromatosis.

\section{References}

1. Listernick R and Charrow J (2004) Neurofibromatosis in childhood. Adv Dermatol 20: 75-115.

2. Grover C, Lohra M, Nanda S, Reddy BS (2005) Leprosy with neurofibromatosis--a diagnostic dilemma. Lepr Rev 76: 91-93.

3. Thomas J, Wilson NC, Parimalam S, Augustine SM, Muthuswami TC (1989) Multiple neurofibromatosis with histoid leprosy. Int J Lepr Other Mycobact Dis57: 695-696.

4. Joseph MS (1985) Von Recklinghausen's disease associated with diffuse lepromatous leprosy--a case report. Indian J Lepr 57: 872-875.

\section{Corresponding author}

Kaliaperumal Karthikeyan

41 Vellala Street

Pondicherry

India

Email: karthikderm@yahoo.co.in

Conflict of interests: No conflict of interests is declared. 